\title{
The Role of Simultaneous Standardized Uptake Value and MRI Diffusion Weighted Heterogeneity Index from Hybrid PET/MR in the Evaluation of Brain Metastases
}

\section{Bahattin Özkul ( $\sim$ bahozkul@yahoo.com)}

Atlas University: Istanbul Atlas Universitesi https://orcid.org/0000-0003-3339-8329

\section{Bedriye Koyuncu Sokmen}

Demiroglu Bilim University: TC Demiroglu Bilim Universitesi

Ibrahim Halil Sever

Demiroglu Bilim University: TC Demiroglu Bilim Universitesi

Nagihan Inan Gurcan

Demiroglu Bilim University: TC Demiroglu Bilim Universitesi

\section{Research Article}

Keywords: ADCCV, Brain, Heterogeneity index, Metastasis, PET/MRI

Posted Date: September 7th, 2021

DOI: https://doi.org/10.21203/rs.3.rs-595957/v2

License: (c) (1) This work is licensed under a Creative Commons Attribution 4.0 International License.

Read Full License

Version of Record: A version of this preprint was published at Eurasian Journal of Medical Investigation on September 1st, 2022. See the published version at https://doi.org/10.14744/ejmi.2022.11286. 


\section{Abstract}

\section{Background}

$\mathrm{PET} / \mathrm{MRI}$ is a hybrid imaging modality and uses for evaluating oncology patients with its benefits of combination of soft tissue contrast and glucose metabolism.

\section{Aims}

To evaluate the role of simultaneous derived apparent diffusion coefficient (ADC) heterogeneity index and 2-[ $\left.{ }^{18} \mathrm{~F}\right]$-fluoro-2-deoxy-o-glucose ( ${ }^{18} \mathrm{~F}$-FDG) standardized uptake value (SUV) from hybrid PET/MRI to differentiate brain metastasis (BM) from normal appearing brain parenchyma (NABP) and to find out the efficiency of combination of $A D C_{C V}$ and SUV against conventional $A D C$ parameter.

\section{Study Design}

Whole-body PET/MRI was performed to evaluate proven 49 BM of 26 oncology patients ( 15 females, 11 males; mean age $63 \pm 16$ years), sourced from different primary cancer.

\section{Methods}

Brain sequences, which were dixon and diffusion weighted imaging (DWI) protocols with simultaneous PET were used to calculate coefficient of variance of the $A D C\left(A D C_{C V}\right)$ and SUVmax. All images were assessed by three radiologists and the same size of VOI was placed on BM and NABP. Inter-rater reliability was tested by inter-class correlation (ICC). The correlation of $A D C_{C V}$ and SUVmax and the differences in ADC values and SUVmax between BM and NABP were investigated.

\section{Results}

The excellent consistency was found between raters at $A D C$ mean (0.972) and $A D C_{C V}$ (0.995). There was a moderate correlation between $\mathrm{ADC}_{\mathrm{CV}}$ and SUVmax $(\mathrm{r}=0.585)$ and a negligible inverse correlation between ADCmean and SUVmax ( $r=-0.154)$. A statistically significant difference between BM and NABP was determined for $A D C_{C V}(p<0.001)$ and SUVmax $(p=0.007)$. An area under the curve (AUC) of 0.663 , $0.966,0.571,0.696$ and 0.971 were obtained with ROC analysis of SUVmax, $A D C_{C V}, A D C m e a n$, SUVmax+ADCmean and SUVmax+ADC $C$, respectively.

\section{Conclusion}

$A D C_{C V}$ may be considered as a new parameter in multi-parametric MRI that quantitatively discriminates BM from NABP with excellent interrater reliability.

\section{Introduction}


Brain metastasis (BM) is associated with poor survival outcomes and poses distinct clinical challenges. Lung cancer, renal cell carcinoma, breast cancer, melanoma and colorectal cancers are the most common causes of BM (1). Due to great variation in imaging appearances, these metastases present a common diagnostic challenge which can affect patient management.

Computed tomography (CT) and magnetic resonance imaging (MRI) are the key imaging modalities used in the diagnosis of BM. In some cases, advanced imaging techniques including proton magnetic resonance spectroscopy (MRS), contrast enhanced magnetic resonance perfusion (MRP), diffusion weighted imaging (DWI), and diffusion tensor imaging (DTI) may help for the diagnosis (2). Although these imaging techniques are essential in the diagnosis, using quantitative data may lead to improved detection of BM.

DWI is a fast, non-contrast MR technique that indicates the random microscopic motion of free water molecules. It is widely appreciated as a qualitative tool in the examination of the central nervous system (CNS). Apparent diffusion coefficient (ADC) is a measure, calculated using DWI and reflects the magnitude of diffusion quantitatively.

Tumors are heterogeneous because of the spatial variation in the cellularity, angiogenesis, extracellular matrix and necrosis $(3,4)$. Higher intratumoral heterogeneity is related with poor prognosis due to its aggressive behavior (5-7). Thus, measuring of tissue heterogeneity may be helpful in the detection of tumors and selection poor prognostic patients for more intensive therapy. There are various methods using complex textural analysis in the detection of tissue heterogeneity (8). Of all these, the coefficient of variation $(\mathrm{CV})$ is easily calculated and shows relative variability. In line with this, $A D C_{C V}$ as a reliable heterogeneity index was used in different studies (9-12).

Positron Emission Tomography (PET)/MRI is a new imaging technology that allows for PET and MRI scans to be acquired simultaneously. Although MRI is the standard neuroimaging technique for detection of tumors and the surrounding anatomical structures in the brain, PET aids to complement MRI in lesion grading, tumor extent delineation and evaluation of the treatment response. Allowing both structural and functional evaluation of tumors in one single scan makes PET/MRI more popular in oncology imaging.

The primary target of our study was to determine the diagnostic performance of $A D C_{C V}$, as a heterogeneity index, to differentiate BM from normal appearing brain parenchyma (NABP), as compared to conventional MRI metrics used in daily routine. A secondary target of this research was to evaluate the efficiency of $A D C_{C V}$ to differentiate $B M$ from NABP when we combined with standard uptake value (SUVmax) simultaneous derived from PET-MRI. To the best of our knowledge, this is the first study that evaluates the diagnostic performance of $A D C_{C V}$ in brain metastases and its correlation with SUVmax on PET/MRI hybrid system.

\section{Material And Methods}

\section{Study Design}


347 consecutive adult patients with known malignancies who underwent PET/MRI between January 2017 and September 2019 were evaluated. Forty-five patients who had BM were enrolled in this retrospective single center study. The patients who has multiple lesions (if there is no enough NABP), a massive brain edema and a history of radiotherapy were excluded from the study. Decision of BM was given if lesions growth at least two imaging methods in the follow-up imaging (3-6 months) or proven with biopsy (single lesion). Thus, 49 lesions of 26 patients were included and analyzed for this study (Fig. 1). All primary malignancies were proven histopathological by biopsy or surgery.

\section{Image acquisition}

Patients fasted at least 6 hours before starting examination and injection of ${ }^{18} \mathrm{~F}-\mathrm{FDG}$ was given if blood glucose levels were $<140 \mathrm{mg} / \mathrm{dL}(7.77 \mathrm{mmol} / \mathrm{L})$. All scans were performed with the patient in the supine position on the 3 Tesla Biograph mMR scanner (Siemens Healthcare, Erlangen, Germany) using a 16channel head and neck surface coil and three 12-channel body coils and the total scanning time was $60 \pm 3$ minutes. The whole-body images, which cover the entire body from head to heel, were obtained in five to six bed positions according to body-mass index (BMI) of patient. PET attenuation correction was performed using four compartment model attenuation map calculated from a Dixon-based volumetric interpolated breath-hold examination (VIBE) sequence. The MRI protocol included sequences as below: T1-weighted slice-selective Turbo Flash (TR/TE, $1600 \mathrm{msec} / 2.5 \mathrm{msec}$ ) in the axial plane, free breath diffusion-weighted imaging using the echo planar imaging technique (EPI) (TR/TE, $12000 \mathrm{msec} / 78$ msec, $b=0 \mathrm{~s} / \mathrm{mm}^{2}$ and $800 \mathrm{~s} / \mathrm{mm}^{2}$ ) in the axial plane and T2-weighted single-shot echo train (HASTE) (TR/TE, $1500 \mathrm{msec} / 87 \mathrm{msec}$ ) in the coronal plane. Contrast enhanced protocol including the breath-hold $3 \mathrm{D}$ VIBE sequence (TR/TE, $4.56 \mathrm{msec} / 2.03 \mathrm{msec}$ ) in the arterial, portal venous and equilibrium phases covering whole-body in the axial plane was performed with using a weight-adapted gadolinium-based contrast agent and all sections were then combined.

\section{Image analysis}

All image datasets were transferred to the dedicated Syngo.via PET/MRI workstation (Siemens Healthcare) and images were assessed separately by three radiologists (i.H.S, B.K.S and N.I.G) with at least 7 years of experience who were blinded to the patients' information. A volume of interest (VOI) was placed manually on axial PET images and all three planes were controlled for ensuring to not overflow the limits of the lesions. The VOI was coregistered and placed on ADC images overlapping with PET images. Manual correction was used to fine tune when the images were not overlapped. For each determined lesion, a similar size of VOI was used on NABP (Fig. 2). Care was taken to keep away from edema, blood vessels and cerebrospinal fluid and for preventing bias, white matter, which did not include sulcus, was used to evaluate NABP. SUVmax (SUV of the hottest voxel within a defined VOI), which is easy to use and operator independent, was calculated automatically and measured on PET images. The mean (ADCmean) and standard deviation (SD) of $\mathrm{ADC}\left(\mathrm{ADC}_{\mathrm{SD}}\right)$ were calculated automatically by software for each measurement. $A D C_{C V}$ was created by dividing the SD by the $A D C$ mean. 


\section{Statistical analysis}

IBM Statistical Package for the Social Sciences (SPSS ver. 21 for windows, Chicago, IL, USA) software was used for all statistical analysis. Intra-class correlation coefficient (ICC) was used for determining inter-rater reliability in $A D C$ mean, $A D C_{S D}$ and $A D C_{C V}$. The ICC value ${ }^{2} 0.50,0.51-0.75,0.76-0.90$ and $>0.90$ were evaluated as indicating poor, moderate, good and excellent reliability, respectively. The fitness of numeric data set to normal distribution was determined by the Shapiro-Wilk test. Due to normal distribution, correlation between SUVmax and $\mathrm{ADC}_{\mathrm{CV}}$ was tested by Pearson correlation. Independent $t$ test was carried out to measure differences between BM and NABP for all variables. Receiver operating characteristics (ROC) analysis based on histopathological results was performed to determine cut-off value, which differentiate BM from NABP, by the Youden index. The area under the curve (AUC), sensitivity and specificity were calculated for each variable. A p-value 0.05 was accepted as statistically significant.

\section{Results}

\section{Patients demographic}

Twenty-six patients ( 15 female, 11 male) with proven BM were included in the study. The patients aged between $28-87$ (mean \pm standard deviation; $63.7 \pm 16.4$ ) years. 20 patients $(77 \%)$ also had metastases in other locations besides the brain. $49 \mathrm{BM}$ were analyzed with VOI mean $3,57 \pm 4,13 \mathrm{~cm}^{3}$. As identified in table 1, breast (invasive ductal carcinoma, 5 patients; invasive lobular carcinoma, 4 patients), lung (adenocarcinoma, 7 patients; small cell carcinoma, 3 patients), colon (adenocarcinoma, 4 patients), kidney (renal cell carcinoma, 2 patients) and skin (malignant melanoma, one patient) were the primary source of tumors for BM.

\section{Interrater reliability}

There was an excellent consistency between raters at $A D C$ mean, $A D C_{S D}, A_{C} C_{C V}$ and SUVmax with ICC 0.972 [ $95 \%$ confidence interval (Cl] 0.952-0.984), 0.990 (95\% Cl 0.983-0.994), 0.995 (95\% Cl 0.992-0.997) and 0.993 (95\% Cl 0.989-0.996), respectively.

\section{Correlation with SUVmax}

For all values, the mean of three raters was calculated and presented as $A D C$ mean, $A D C_{C V}$ and SUVmax. According to Pearson correlation coefficient, there was a moderate positive correlation $(r=0.585, p<0.000)$ between $A D C_{C V}$ and SUVmax when all measurements included (BM+NABP). A negligible inverse correlation was found between ADCmean and SUVmax $(r=-0.154, p=0.044)$.

\section{Differences between $B M$ and NABP}

A statistically significant difference between BM and NABP with $p<0.001$ and $=0.007$ value was found for $A D C_{C V}$ and SUVmax, respectively. There was no statistically significant difference for ADCmean 
$(p=0.076)$. The mean $\pm S D$ values of $A D C$ mean, $A D C_{C V}$ and $S U V m a x$ of all lesions were presented in the table (table 2).

The cut-off value based on the maximum Youden index to determine differentiation between BM and NABP was ${ }^{3} 3.34$ for SUVmax, $0.84 \times 10^{-3} \mathrm{~mm}^{2} / \mathrm{s}$ for ADCmean and ${ }^{3} 0.08$ for $\mathrm{ADC}_{\mathrm{CV}}$. An AUC for SUVmax

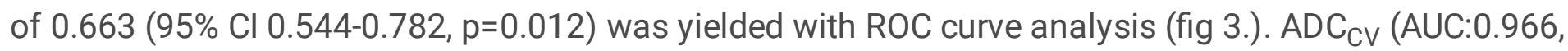
$p<0.001)$ had higher AUC with a smaller standard error and a narrower confidence interval than ADCmean (AUC: $0.571, p=0.273$ ) and SUVmax (Fig. 3). The sensitivity and specificity of $A D C_{C V}(82.5 \%, 97.5 \%$, respectively) were higher than SUVmax $(67.5 \%, 60 \%$, respectively) and ADCmean (55\%, $70 \%$, respectively). When we combined SUVmax and ADCmean to discriminate BM and NABP, the AUC was $0.696(95 \% \mathrm{Cl}$ 0.581-0.810) yielding a better sensitivity (70\%). The highest AUC (0.971) was found in combination of ADCcv and SUVmax with a sensitivity of $97.5 \%$ and specificity of $87.5 \%$ (Fig. 4). AUC, sensitivity, specificity, confidence interval and standard error of all values were summarized in the table (table 3 ).

\section{Discussion}

In this study, we investigated the role of $A D C_{C V}$ derived from PET/MRI as a heterogeneity index in discriminating $B M$ from NABP. The main finding of this study was that, $A D C_{C V}$ is more effective to discriminate $B M$ from NABP compared to conventional $A D C$ parameters. Besides, $A D C_{C V}$ had higher potential to discriminate BM from NABP when we combined with SUVmax from simultaneous derived $\mathrm{PET} / \mathrm{MRI}$ system.

Integrated PET / MRI scanners with the recent developments, new opportunities have emerged for quantitative molecular imaging. PET / MRI provides multimodal analysis of concurrently acquired functional parameters that can contribute to better characterization of tumor biology and also help identify markers to predict response to therapy $(13,14)$.

Due to the high ${ }^{18} \mathrm{~F}-\mathrm{FDG}$ uptake of the cerebral cortex and the low spatial resolution of PET imaging, FDG $\mathrm{PET} / \mathrm{CT}$ imaging has limitations, especially in the detection of small metastases in the brain. Sensitivity of FDG PET/CT in brain imaging is low. In retrospective comparative studies, it is stated that FDG PET/CT imaging at the time of diagnosis can capture up to $61 \%$ of metastatic brain lesions that can be detected by MRI (15). Therefore, PET / MR imaging may be preferred in brain metastasis scanning because of the high soft tissue contrast of MR imaging.

SUVmax measured by PET and ADC measured by MRI allow assessment of water diffusion and glucose metabolism in tumor cells. The results of the present study show that $A D C_{C V}$ exhibits an improved correlation with SUVmax. Moreover, it provides better quantitative separation between BM and NABP, as compared to common MRI metrics.

In this study, $A D C m e a n$ showed a significant negative correlation with SUVmax; however, $A_{C D} C_{C V}$ showed higher correlation with SUVmax than ADCmean parameter. There are previously reported oncologic 
studies of the inverse correlation found between ADC and SUV. Several of these studies reported significant strong inverse correlation between ADCmean and SUVpeak in rectal cancer (16), a significant inverse correlation between ADCmean and SUVmean in gastrointestinal stromal tumor (17), and recently an inverse correlation between ADC and PET SUV in liver tumors (18).

Tumor heterogeneity consists of marked differences in cell mix, size, and arrangement. Heterogeneity also plays a role in micro-environmental factors (including oxygenation, $\mathrm{pH}$, interstitial pressure, blood flow), metabolism, and gene expression. This deep heterogeneity is extremely important for prognosis, treatment planning, and drug distribution, which ultimately affects patient outcomes. There are a number of ways to investigate tumor heterogeneity, some of which include functional and molecular imaging, which can be applied to clinical data (19).

The characterization of tissues can be improved using histogram-based assessments of the distribution of $A D C$ values. Histogram approaches have multiple advantages, including volume-of-interest (VOI) assessments, thus avoiding the subjectivity that is inherent with ROI placements. Importantly, histograms can provide additional metrics that reflect the texture of lesions, thereby allowing heterogeneity of ADC distribution within tissue to be assessed (20). Tissue heterogeneity analysis is rapidly evolving by various methods. Despite most of the tools currently offered are often complex and computationally costly, it is an easy to calculate texture parameter of $A D C_{C V}$. Several studies have used $C V$ as an index of heterogeneity in recent years.

In a study in liver metastasis, the results of this study show that $A D C_{C V}$ can significantly distinguish between liver metastasis and normal-appearing liver (9). Similar to our study, there was a good correlation between $A D C_{C V}$ and SUV peak in this study. Significant differences in CV diffusion index was found in another study about hepatocellular carcinoma in fresh liver explants (21).

PET / CT and DWI share applications in clinical oncology. While both SUV and ADC correlate with cellularity, SUV is also associated with several other pathological markers such as mitotic count, presence or absence of necrosis (22). For this reason, PET / MRI oncological evaluation is also valuable when these two parameters (SUV and ADC) are obtained together in the same examination. In a study by Nakajo et al. (23), 44 patients with breast cancer underwent preoperative PET/CT and DWI within an average of 17 days between examinations, and both SUVmax and ADC were significantly associated ( $p<$ 0.05 ) with histologic grade (independently), nodal status, and vascular invasion. This finding suggests that SUVmax and ADC correlate with several of the pathologic prognostic factors and that both values may have the same potential for being predictive of the prognosis of breast cancer.

In oncology, imaging has a very important place in evaluating response to treatment. For this reason, many studies are aimed at understanding the structure and heterogeneity of the tumor. Therefore, it is essential to develop quantitative imaging methods and objective biomarkers to improve the diagnosis of brain metastasis. As a volume-independent index of heterogeneity, $\mathrm{ADC}_{\mathrm{CV}}$ can be considered as a 
potential biomarker that quantitatively differentiates BM from NABP. Tissue heterogeneity has been proposed as a basis for a biomarker for tumors $(3,4,24)$.

This hybrid PET / MRI study shows a significant negative correlation between metabolic activity on ${ }^{18} \mathrm{~F}$ FDG PET and water diffusion over DWI in brain metastasis, possibly because both parameters are directly related to tumor cellularity. The correlation found between SUVs and $A D C$ mean, $A D C_{C V}$ values supports the idea that high cellularity due to tumor proliferation results in greater metabolic activity and restricts water diffusion.

Nowadays, using multi-parametric brain MRI (MR spectroscopy, MR perfusion, DWI, routine contrast enhanced MRI) to evaluate cranial pathologies becomes a routine practice. We think that ADCcv has a potential to evaluate tumor heterogeneity and may be a new parameter in multi-parametric MRI. Studies on differentiating local recurrence in brain tumor and radiotherapy associated changes or tumor and other cranial pathologies (brain abscess, leukodsytrophy, lymphoma, etc) may support our hypothesis.

This study has several limitations. First, this was a retrospective study and performed on a relatively small study population. Another limitation was the difficulty in determining the limits of the lesions due to the limited resolution of PET. The accuracy of the results obtained from our study should be supported by using different software in larger patient groups and with multi-center studies. The last limitation of our study was that brain metastases originated from different sources.

\section{Conclusions}

In conclusion, using PET/MRI instead of PET/CT decreases radiation dose, however radiation exposure caused by short term follow-up imaging of oncology patients continue to be an issue. Although determining brain metastases compared to normal brain parenchyma are not the main challenge in oncologic patients, $A D C_{C V}$ may be helpful to clinicians for avoiding further radiation exposure of patients and for managing patients when using contrast media is contraindicated. Moreover, it would be easy to implement $A D C_{C V}$ in a clinical setting. Future studies that will blindly and independently identify $B M$ in NABP using PET ${ }^{18} \mathrm{~F}-\mathrm{FDG}$ SUV and $D W I A D C_{C V}$ will present potential to investigate $A D C_{C V}$ as a new parameter for BM.

\section{References}

1. Kumar V, Abbas AK, Fausto N, Robbins SL. Robbins and Cotran Pathologic Basis of Disease: Elsevier Saunders; 2005.

2. Fink KR, Fink JR. Imaging of brain metastases. Surg Neurol Int. 2013;4(Suppl 4):S209-19.

3. Boroughs LK, DeBerardinis RJ. Metabolic pathways promoting cancer cell survival and growth. Nat Cell Biol. 2015;17(4):351-9. 
4. Robertson-Tessi M, Gillies RJ, Gatenby RA, Anderson AR. Impact of metabolic heterogeneity on tumor growth, invasion, and treatment outcomes. Cancer Res. 2015;75(8):1567-79.

5. Ganeshan B, Miles KA, Young RC, Chatwin CR. Hepatic entropy and uniformity: additional parameters that can potentially increase the effectiveness of contrast enhancement during abdominal CT. Clin Radiol. 2007;62(8):761-8.

6. Ganeshan B, Abaleke S, Young RC, Chatwin CR, Miles KA. Texture analysis of non-small cell lung cancer on unenhanced computed tomography: initial evidence for a relationship with tumour glucose metabolism and stage. Cancer Imaging. 2010;10(1):137-43.

7. Miles KA, Ganeshan B, Griffiths MR, Young RC, Chatwin CR. Colorectal cancer: texture analysis of portal phase hepatic CT images as a potential marker of survival. Radiology. 2009;250(2):444-52.

8. Alic L, Niessen WJ, Veenland JF. Quantification of heterogeneity as a biomarker in tumor imaging: a systematic review. PLoS One. 2014;9(10):e110300.

9. Stein D, Goldberg N, Domachevsky L, Bernstine H, Nidam M, Abadi-Korek I, et al. Quantitative biomarkers for liver metastases: comparison of MRI diffusion-weighted imaging heterogeneity index and fluorine-18-fluoro-deoxyglucose standardised uptake value in hybrid PET/MR. Clin Radiol. 2018;73(9):832.e17-.e22.

10. Dong X, Wu P, Sun X, Li W, Wan H, Yu J, et al. Intra-tumour 18F-FDG uptake heterogeneity decreases the reliability on target volume definition with positron emission tomography/computed tomography imaging. J Med Imaging Radiat Oncol. 2015;59(3):338-45.

11. Yoon HJ, Kim Y, Kim BS. Intratumoral metabolic heterogeneity predicts invasive components in breast ductal carcinoma in situ. Eur Radiol. 2015;25(12):3648-58.

12. Bundschuh RA, Dinges J, Neumann L, Seyfried M, Zsótér N, Papp L, et al. Textural Parameters of Tumor Heterogeneity in ${ }^{1}$ F-FDG PET/CT for Therapy Response Assessment and Prognosis in Patients with Locally Advanced Rectal Cancer. J Nucl Med. 2014;55(6):891-7.

13. Yankeelov TE, Peterson TE, Abramson RG, Izquierdo-Garcia D, Arlinghaus LR, Li X, et al. Simultaneous PET-MRI in oncology: a solution looking for a problem? Magn Reson Imaging. 2012;30(9):1342-56.

14. Punwani S, Taylor SA, Saad ZZ, Bainbridge A, Groves A, Daw S, et al. Diffusion-weighted MRI of lymphoma: prognostic utility and implications for PET/MRI? Eur J Nucl Med Mol Imaging. 2013;40(3):373-85.

15. Rohren EM, Provenzale JM, Barboriak DP, Coleman RE. Screening for cerebral metastases with FDG PET in patients undergoing whole-body staging of non-central nervous system malignancy. Radiology. 2003;226(1):181-7. 
16. Wong CS, Gong N, Chu Y-C, Anthony M-P, Chan Q, Lee HF, et al. Correlation of measurements from diffusion weighted MR imaging and FDG PET/CT in GIST patients: ADC versus SUV. European Journal of Radiology. 2012;81(9):2122-6.

17. van Rijswijk CSP, Geirnaerdt MJA, Hogendoorn PCW, Peterse JL, van Coevorden F, Taminiau AHM, et al. Dynamic contrast-enhanced MR imaging in monitoring response to isolated limb perfusion in highgrade soft tissue sarcoma: initial results. European radiology. 2003;13(8):1849-58.

18. Kong E, Chun KA, Cho IH. Quantitative assessment of simultaneous F-18 FDG PET/MRI in patients with various types of hepatic tumors: Correlation between glucose metabolism and apparent diffusion coefficient. PloS one. 2017;12(7):e0180184-e.

19. Just $\mathrm{N}$. Improving tumour heterogeneity MRI assessment with histograms. $\mathrm{Br} \mathrm{J}$ Cancer. 2014;111(12):2205-13.

20. Dalili D, Padhani A, Grimm R. Quantitative WB-MRI with ADC Histogram Analysis for Response Assessment in Diffuse Bone Disease. 2017.

21. Rosenkrantz AB, Sigmund EE, Winnick A, Niver BE, Spieler B, Morgan GR, et al. Assessment of hepatocellular carcinoma using apparent diffusion coefficient and diffusion kurtosis indices: preliminary experience in fresh liver explants. Magn Reson Imaging. 2012;30(10):1534-40.

22. Rakheja R, Makis W, Skamene S, Nahal A, Brimo F, Azoulay L, et al. Correlating metabolic activity on 18F-FDG PET/CT with histopathologic characteristics of osseous and soft-tissue sarcomas: a retrospective review of 136 patients. AJR Am J Roentgenol. 2012;198(6):1409-16.

23. Nakajo M, Kajiya Y, Kaneko T, Kaneko Y, Takasaki T, Tani A, et al. FDG PET/CT and diffusionweighted imaging for breast cancer: prognostic value of maximum standardized uptake values and apparent diffusion coefficient values of the primary lesion. Eur J Nucl Med Mol Imaging. 2010;37(11):2011-20.

24. Marusyk A, Polyak K. Tumor heterogeneity: causes and consequences. Biochim Biophys Acta. 2010;1805(1):105-17.

\section{Tables}

Table 1. Primary source and histopathology of metastases and distribution of the study population. 


\begin{tabular}{llrr}
\hline Primary Source & Histopathology & No. of patients & No. of metastases \\
& & 5 & 11 \\
\hline Breast & Invasive ductal & 4 & 9 \\
& Invasive lobular & 7 & 9 \\
Lung & Adenocarcinoma & 3 & 5 \\
& Small cell carcinoma & 4 & 6 \\
Colon & Adenocarcinoma & 2 & 3 \\
Kidney & Renal cell carcinoma & 1 & 6 \\
Skin & Malignant melanoma & 26 & 49
\end{tabular}

Table 2. A comparison between SUV and ADC data according to mean of three raters in normal appearing brain parenchyma and brain metastases.

\begin{tabular}{lcccr}
\hline Variable (n=40) & $\begin{array}{l}\text { Normal } \\
\text { parenchyma }^{\mathbf{a}}\end{array}$ & appearing & $\begin{array}{c}\text { brain } \\
\text { Brain } \\
\text { metastases }^{\mathbf{a}}\end{array}$ & $\begin{array}{c}\text { p-two } \\
\text { tailed }^{\mathbf{b}}\end{array}$ \\
\hline SUVmax & $3.19 \pm 0.55$ & $3.51 \pm 0.49$ & 0.007 \\
$\begin{array}{l}\mathrm{ADCmean} \\
\left.\mathrm{mm}^{2} / \mathrm{s}\right)\end{array}$ & $\left(10^{-3}\right.$ & $0.82 \pm 0.07$ & $0.86 \pm 0.15$ & 0.076 \\
$\mathrm{ADC}_{\mathrm{CV}}$ & & & & \\
\hline
\end{tabular}

*SUV, standardized uptake value; ADC, apparent diffusion coefficient; CV, coefficient of variance

a mean \pm Standard deviation

${ }^{\mathrm{b}}$ Independent $t$ test

Table 3. Receiver operating characteristics analysis

\begin{tabular}{lclcccr}
\hline Variable & AUC & SE & $\begin{array}{c}\text { 95\% Confidence } \\
\text { interval }\end{array}$ & Sensitivity & Specificity & p \\
\hline SUV & 0.663 & 0.061 & $0.544-0.782$ & $67.5 \%$ & $60 \%$ & .012 \\
ADCmean & 0.571 & 0.069 & $0.436-0.706$ & $55 \%$ & $70 \%$ & .273 \\
ADC $_{\text {CV }}$ & 0.966 & 0.018 & $0.931-1.000$ & $82.5 \%$ & $97.5 \%$ & $<.001$ \\
SUV + & 0.696 & 0.058 & $0.581-0.810$ & $70 \%$ & $65 \%$ & .003 \\
ADCmean & & & $0.940-1.000$ & $97.5 \%$ & $87.5 \%$ & $<.001$ \\
\hline
\end{tabular}

*AUC, area under the curve; SE, standard error; SUV, standardized uptake value; ADC, apparent diffusion coefficient; $\mathrm{CV}$, coefficient of variance

\section{Declarations}




\section{Funding:}

This research did not receive any specific grant from funding agencies in the public, commercial, or notfor-profit sectors.

\section{Conflict of interest:}

The authors have no relevant financial or non-financial interests to disclose.

\section{Ethics approval:}

This study was performed in line with the principles of the Declaration of Helsinki. Approval was granted by the Ethics Committee of Demiroğlu Bilim University (Date. 27.10.2020/No. 2020-20-04).

\section{Consent to participate:}

Informed consent was obtained from all individual participants included in the study.

\section{Consent for publication:}

Patients signed informed consent regarding publishing their data.

\section{Availability of data and material:}

The datasets analyzed in the current study are available from the corresponding author on reasonable request.

\section{Code availability:}

Syngo.via (Material no:10496180, Serial no:130408)

IBM SPSS V.21 (Authorization code: 4b37d8fed81bc545db11)

\section{Author's contribution:}

Conceptualization: [Bahattin Özkul]; Methodology: [Bahattin Özkul], [Bedriye Koyuncu Sökmen]; Formal analysis and investigation: [Bahattin Özkul], [ibrahim Halil Sever], [Bedriye Koyuncu Sökmen]; Writing - 
original draft preparation: [Bahattin Özkul], [Bedriye Koyuncu Sökmen], [ibrahim Halil Sever]; Writing review and editing: [Bahattin Özkul], [Nagihan İnan Gürcan]; Supervision: [Nagihan İnan Gürcan].

\section{Figures}

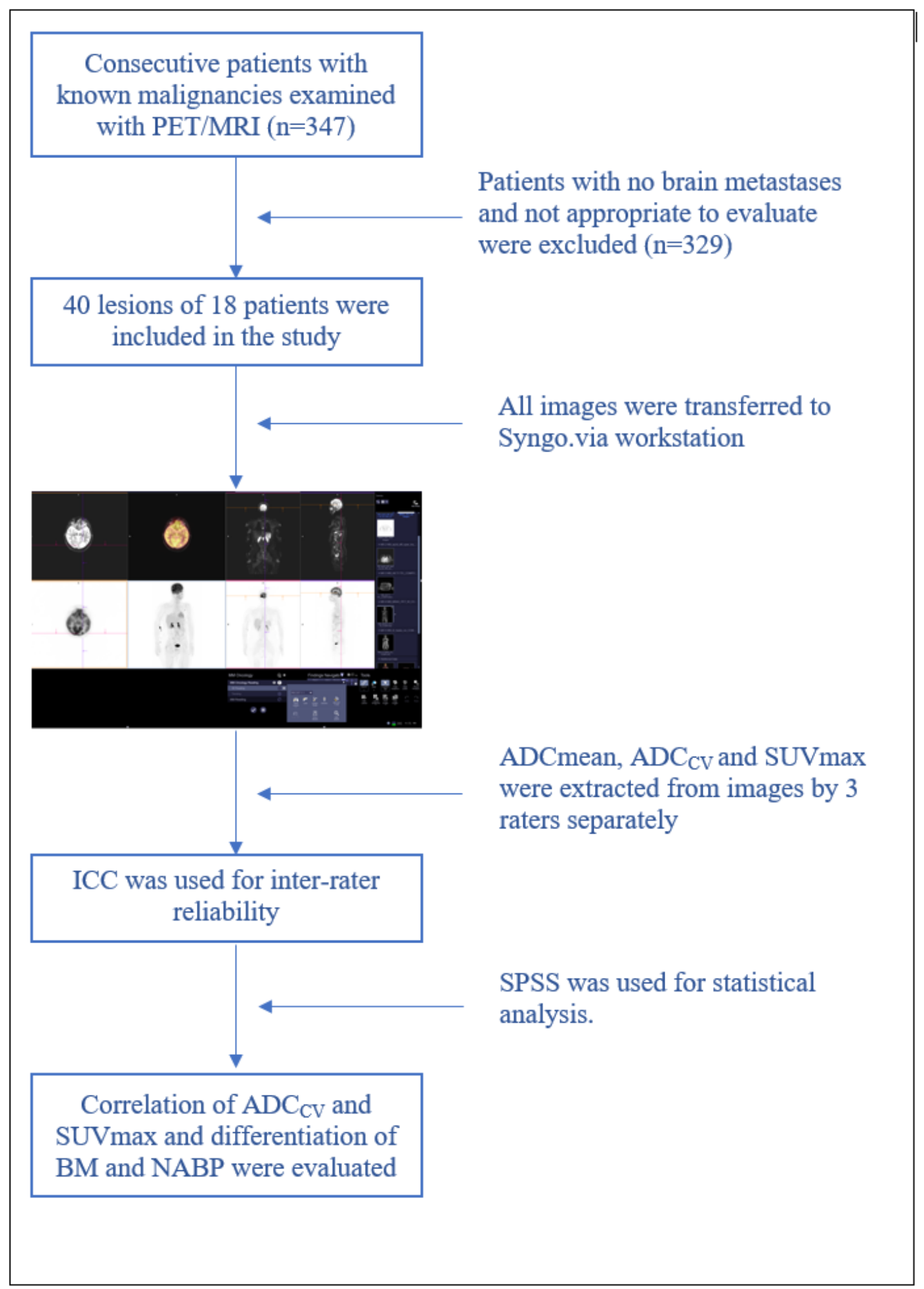

Figure 1 
Design of the study * ADC, Apparent diffusion coefficient; CV, coefficient of variation; SUV, Standardized uptake value; ICC, Inter-class correlation coefficient; SPSS, Statistical package for the social sciences; BM, Brain metastases; NABP, Normal appearing brain parenchyma
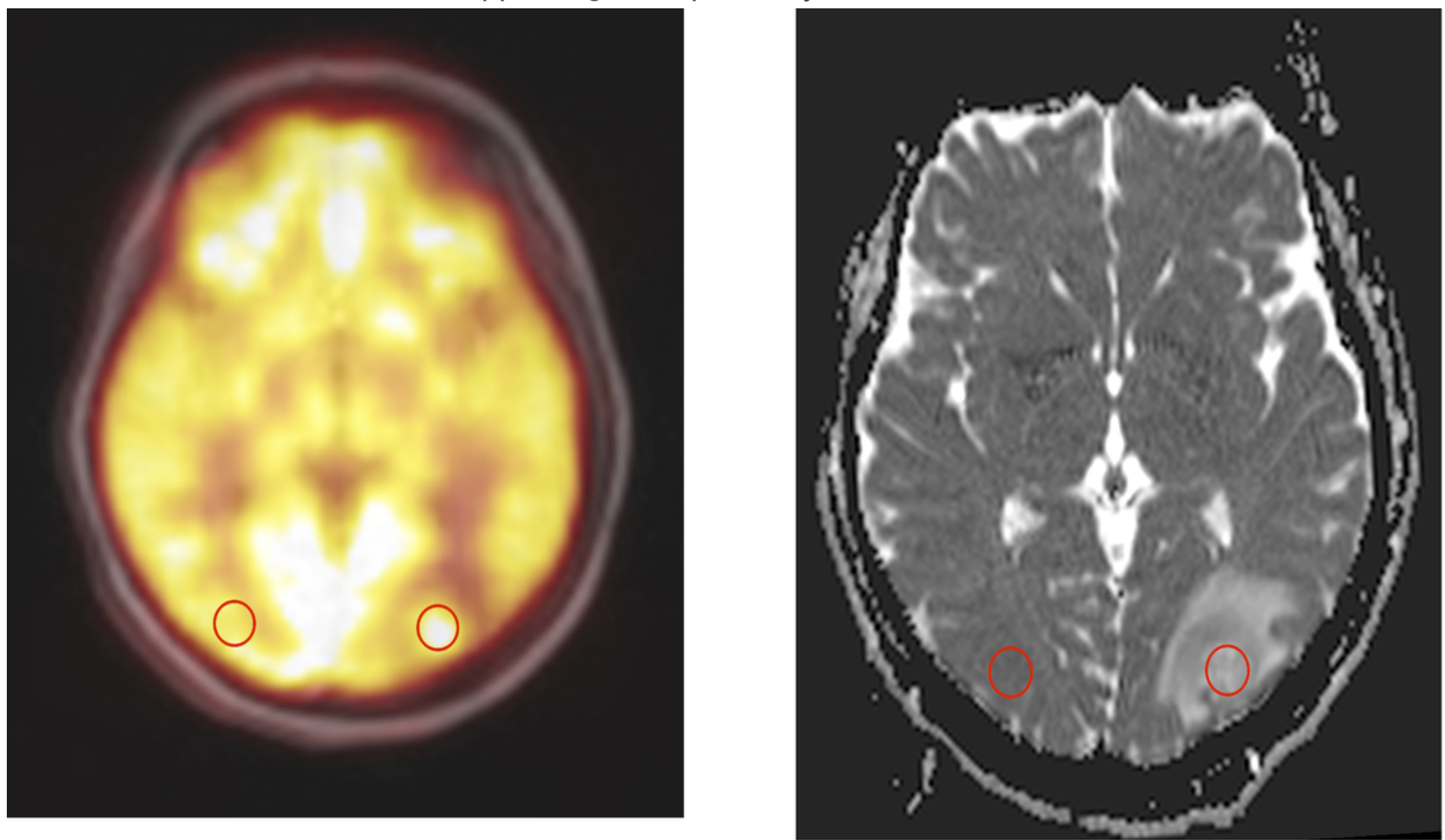

\section{Figure 2}

a-b. Replacing the VOI a) Two VOls with same size was drawn on brain metastasis and normal appearing brain parenchyma with giving care to tumor margins on axial SUV-PET images b) Both VOI was copied and placed with the same location on axial ADC images
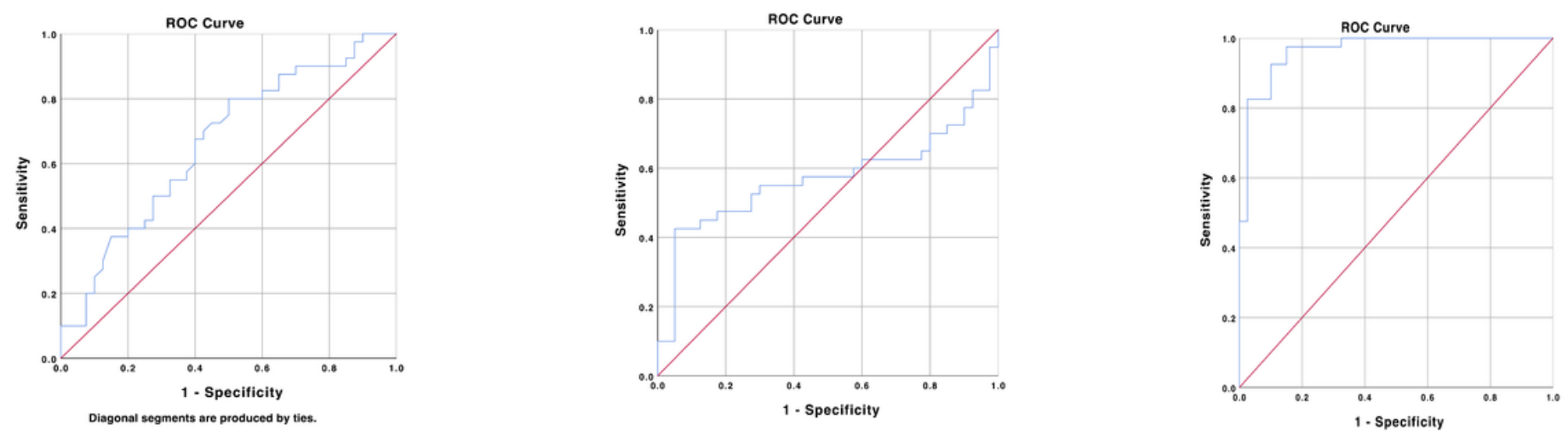

Figure 3 
a-c. Receiver operating characteristics (ROC) curve and area under the curve (AUC) of a) SUVmax, b) $A D C m e a n$ and c) ADCcv to discriminate brain metastasis and normal appearing brain parenchyma
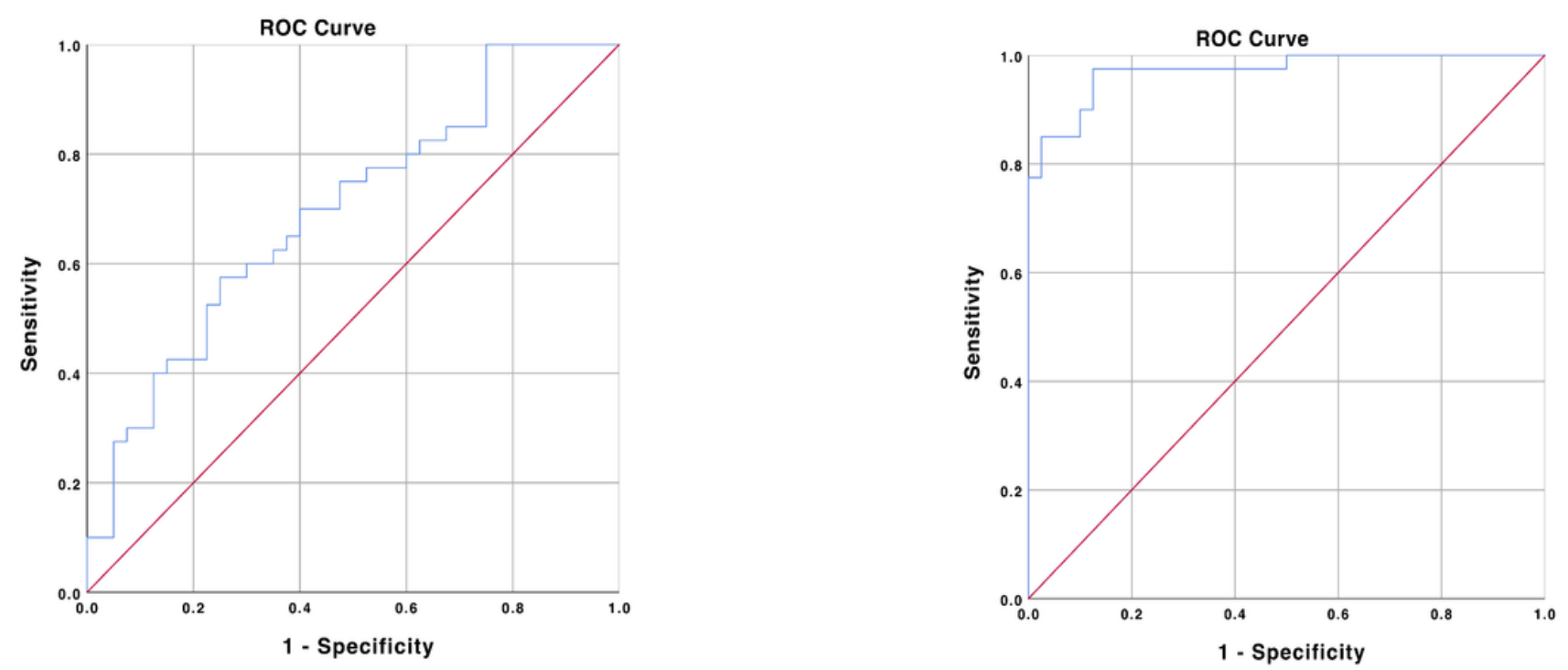

Figure 4

a-b. Receiver operating characteristics (ROC) curve and area under the curve (AUC) of a) SUVmax + ADCmean, b) SUVmax + ADCcv to discriminate brain metastasis and normal appearing brain parenchyma 